\title{
Profils territoriaux de l'évolution de la population du Saguenay-Lac-Saint-Jean, 1951-2001
}

\author{
Majella-J. Gauthier, Éric Tremblay et Carl Brisson ${ }^{1}$
}

\section{Introduction}

Le but de cette analyse est de présenter un portrait de l'évolution des effectifs de la population dans toutes les municipalités qui composent la région du Saguenay-Lac-Saint-Jean sur une longue période, soit cinquante ans. Il s'agit également de voir comment la nouvelle ville de Saguenay tire son épingle du jeu. Ainsi, on peut se demander s'il y a des différences sensibles d'une localité à l'autre, s'il y a des similitudes favorisant la formation d'une typologie, s'il y a une distribution spatiale épousant un patron original; aussi, on peut s'interroger si Saguenay, le château fort régional, avec ses 148250 habitants, y joue un rôle primordial.

Il faut dire que, selon Oris et al., la «connaissance des faits démographiques d'hier et d'aujourd'hui, dans leur dimension spatiale, doit contribuer à construire un outil d'analyse indispensable à l'aide à la décision et à l'élaboration de politiques de développement durable pour notre société $»^{2}$. Il faut ajouter également que la variable population, comme actrice et comme dénominateur, est centrale. Dans une conception plus pragmatique, il est obligatoire d'intégrer la démographie à tous les niveaux de découpage spatial pour assurer des choix politiques favorisant le développement ou la création d'un environnement favorable à l'épanouissement de la société.

Dans le cas qui nous intéresse, il ne s'agit pas uniquement de présenter les faits, mais aussi d'apporter des pistes d'explication et de compréhension. Il y des raisons historiques (économiques, démographiques, de migration, politiques), certaines sont davantage d'ordre géographique (situation, environnement physique), alors que d'autres sont liées à l'organisation de l'espace (proximité, distances, organisation urbaine).
Notre réflexion est présentée en trois points : un portrait régional de l'évolution des effectifs de population dans les territoires locaux, la situation de Saguenay dans la dynamique, et ce que réserve l'avenir.

\section{Un portrait régional des territoires locaux}

\section{Bref historique}

La colonisation de la région du Saguenay-Lac-SaintJean débute en 1838 avec l'arrivée des premiers colons en provenance de Charlevoix; c'est donc un territoire jeune. Rapidement, la hache, le godendart et la charrue deviennent les instruments fondamentaux de la prise de possession du territoire régional. Au rythme des saisons, la forêt et les champs poussent l'étalement de la population le long de la rivière Saguenay jusqu'au Lac-Saint-Jean; cela est vu comme la première vague de peuplement. Puis, c'est la continuation de l'occupation des terres autour du lac Saint-Jean entre 1880 et 1915 , soit la deuxième vague de peuplement. Une troisième poussée a amené des populations à conquérir de nouveaux espaces dans l'arrière-pays entre 1930 et $1940^{3}$.

L'industrialisation de la région à la fin $\mathrm{du} \mathrm{XIX}^{\mathrm{e}}$ siècle et l'arrivée des pulperies marquent une étape importante de l'histoire. L'expansion remarquable, à partir des années 1920, du secteur des pâtes et papiers et de la production de l'aluminium change irrévocablement l'économie régionale. À partir de la décennie 1950, le contexte mondial de l'après-guerre favorise un développement socio-économique spectaculaire. Toutefois, un ralentissement brutal s'installe après la crise économique mondiale des années 1980. 


\section{Méthodologie}

D'entrée de jeu, abordons quelques précisions méthodologiques particulières, En effet, il n'est pas toujours facile de décrire en même temps et d'une manière simple la variation des effectifs de la population de plusieurs collectivités. Cela est d'autant plus un défi lorsque les données correspondent à une série de recensements étalés sur une longue période. Il faut dire cependant que, plus souvent qu'autrement, les études ne tiennent compte que des modifications qui se sont exercées entre les deux années les plus éloignées (les deux termes); cette façon de faire indique une variation simple et générale en effectifs et/ou en pourcentage. Cela est insuffisant puisqu'on sait très bien que des modulations peuvent intervenir, qu'il peut y avoir des périodes de gains ou de pertes et des périodes de stabilité à l'intérieur d'une même collectivité.

La méthode qui a été utilisée ici repose essentiellement sur la confection d'une typologie réalisée à partir d'une classification de diagrammes qui décrivent simplement la variation des effectifs de la population de chacune des 56 municipalités (subdivisions de recensement). Cette méthode s'inspire du traitement des courbes mis au point par Jacques Bertin ${ }^{4}$.

Les données proviennent des divers recensements du Canada (à tous les cinq ans), soit de 1951 à 2001. À ce propos, nous avons été très stricts quant à l'emploi de ces chiffres. Ainsi, seuls les chiffres officiels du recensement ont été employés. La comparaison des entités spatiales a été effectuée en utilisant le découpage du territoire tel que l'a fait le Recensement du Canada de 2001. Ainsi, il est plus approprié de dire que l'on a affaire à des territoires locaux plutôt qu'à des municipalités (par exemple, Saguenay n'existait pas en 2001, et encore moins en 1951).

Les diagrammes donnent une image détaillée des effectifs de la population pour les onze années de recensement. Afin de définir une forme simple, une silhouette de ce qui se passe sur les cinquante années, nous avons procédé à une généralisation mathématique. Celle-ci a été réalisée en exécutant une régression polynomiale du deuxième degré; elle résume en une seule courbe, dite courbe générale (un seul arc), la variation des effectifs de la population. Les dia- grammes originaux pour chaque municipalité sont accessibles directement sur le site Internet de l'Atlas en cliquant sur la municipalité pour laquelle on désire avoir plus de détails (www.uqac.ca/atlas) ${ }^{5}$.

\section{Une typologie éclairante}

La carte 1 est une synthèse de l'analyse des courbes généralisées. Elle montre des profils, des formes de courbes, qui ont été révélés par les diagrammes. Huit groupements de profils différents se dégagent, chacun rassemblant des courbes qui s'apparentent. Les différents profils qui apparaissent sur la carte montrent d'abord deux choses : la variation des effectifs et le sens des taux de variation. S'ajoutent à cela des localisations géographiques privilégiées, c'est-à-dire qui s'organisent dans l'espace.

\section{Les diagrammes originaux pour chaque municipalité sont accessibles directement sur le site Internet de l'Atlas en cliquant sur la municipalité pour laquelle on désire avoir plus de détails (www.uqac.ca/atlas).}

Profil $n^{\circ} 1$ : augmentation de la population et taux de variation positif. Ce type de profil concerne 10 municipalités et touche $9,2 \%$ de la population de 2001, soit 25714 personnes. À ces endroits, l'augmentation de la population s'est vraiment fait sentir au cours des derniers recensements; par exemple, elle a crû de $50 \%$ de 1976 à 2001. On y trouve principalement les municipalités périurbaines autour des centres urbains du Haut-Saguenay telles que Saint-Honoré (voir le diagramme A). Au Lac-Saint-Jean, elles sont localisées aussi en périphérie des centres urbains, sauf autour de Dolbeau-Mistassini. Soulignons que, dans certains cas, l'attrait des ressources aquatiques s'additionne à la proximité urbaine; c'est ce qui se passe par exemple à Laterrière, à Lac-Kénogami et à SaintGédéon.

Profil $n^{\circ} 2$ : augmentation de la population et taux de variation relativement constant. Ce type de profil apparaît uniquement dans la couronne périurbaine au nord de Jonquière et de Chicoutimi. Seules Shipshaw et Saint-David-de-Falardeau font partie de cet ensemble; elles regroupent 1,9\% des habitants de 2001. 


\section{Carte 1 - Profils de l'évolution de la population des territoires}

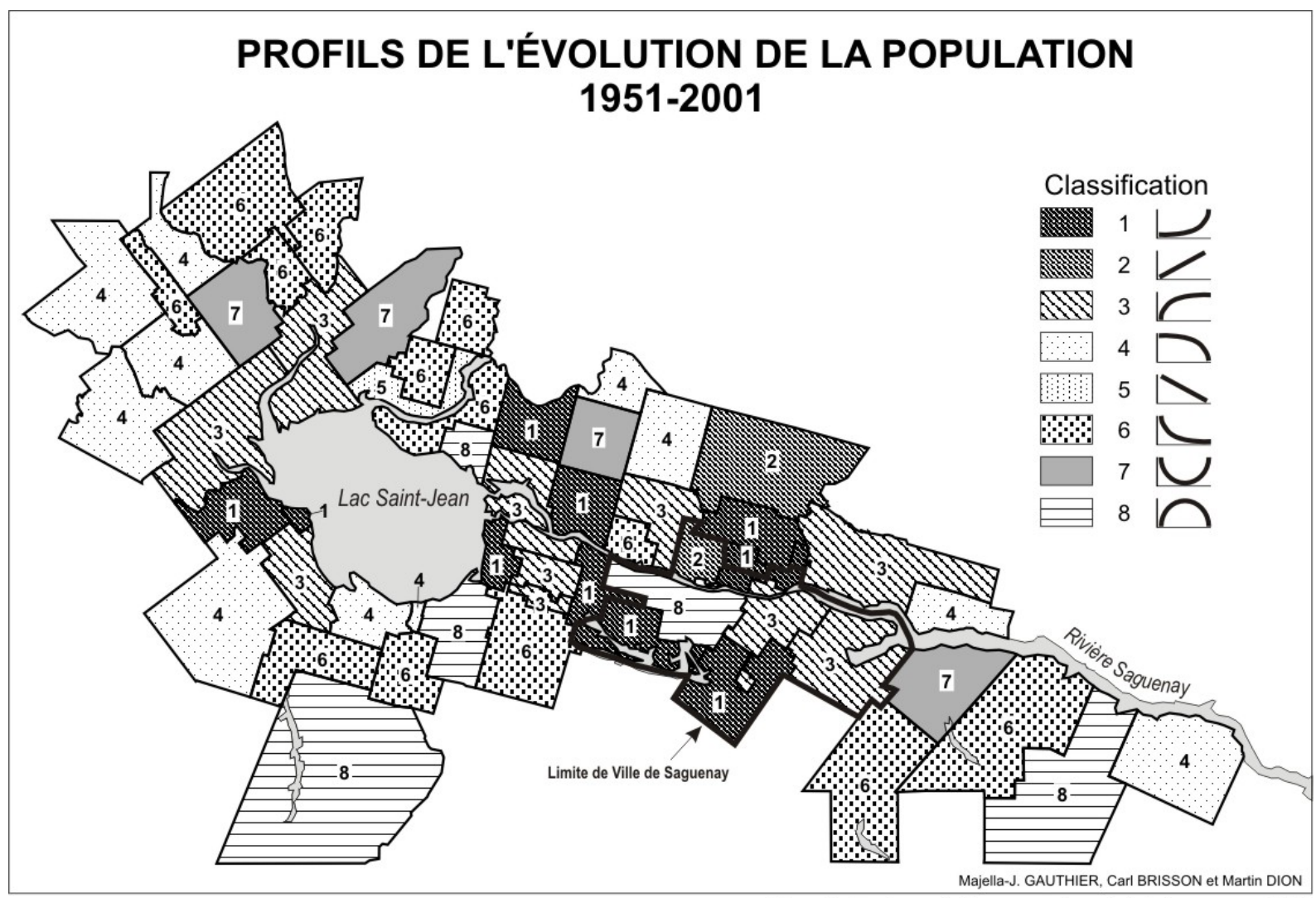

Atlas électronique du Saguenay-Lac-Saint-Jean, mars 2004

Profil $n^{\circ} 3$ : augmentation de la population et taux de variation négatif. Dix municipalités épousent la forme de ce type. Les centres urbains, sauf Jonquière, ont le même comportement: montée fulgurante dans la première moitié de la période, puis maintien ou légère baisse vers la fin. Ailleurs, dans la majorité des cas, ce sont des municipalités qui ne sont pas très éloignées des centres urbains du Haut-Saguenay et du sud d'Alma. Ajoutons que la nouvelle ville de Saguenay et l'ensemble de la région épousent aussi ce profil (voir le diagramme B). C'est le profil le plus populeux avec 155661 habitants en 2001, soit $56 \%$ de la région.

Le profil $n^{\circ} 4$ : baisse de la population et taux de variation négatif. On compte 11 municipalités dans ce groupe. Ici, les effectifs de la population se maintiennent assez élevés au début de la période, mais déclinent rapidement dans la deuxième partie. Elles ont perdu $15 \%$ de leurs habitants au cours des 25 dernières années. Les municipalités qui empruntent ce comportement correspondent à des espaces ruraux situés à la périphérie de la région; elles sont davantage présentes au Lac-Saint-Jean, comme par exemple à Chambord (voir le diagramme C), bien que le BasSaguenay en compte deux. Ce profil rassemble 4,9\% de la population de 2001 .

Profil $n^{\circ} 5$ : baisse de la population et taux de variation relativement constant. Seule Péribonka fait partie de ce groupe $(0,2 \%$ de la population régionale).

Profil $n^{\circ} 6$ : baisse de la population et taux de variation positif. Il y a 13 municipalités qui épousent ce profil et elles comptent 9399 personnes en 2001 (3,4\% de la région). À ces endroits, la chute de la population s'est fait sentir dès le début de la période, pour se maintenir à un niveau plus bas au cours des derniers recensements. Signalons que la baisse de la population fut de $20 \%$ entre 1951 et 1976 . Les municipalités ayant ce comportement se localisent généralement à la marge de l'écoumène et partagent un mode de distribution spatiale sensiblement pareil au profil $n^{\circ} 4$. On remarque cependant une concentration plus 
grande de ce type au nord du Lac-Saint-Jean. SaintStanislas en est un bon exemple (voir diagramme D).

Profil $n^{\circ} 7$ (en creux) : baisse de la population suivie d'une augmentation, avec taux de variation positif. Cinq municipalités sont comprises dans cette classe. Ici, la population a décru au début de la période, mais a subi une augmentation lors des derniers recensements. On y trouve certaines municipalités rurales accolées à des centres urbains tels Dolbeau-Mistassini et La Baie. Le cas de Saint-Félix-d'Otis mérite d'être souligné. Cette petite municipalité avait, au début de la période, toutes les caractéristiques des espaces ruraux peu dynamiques, mais depuis quelque temps, elle s'est transformée à la fois en milieu périurbain et en lieu de résidence dans un environnement naturel (présence de nombreux lacs et de la rivière Saguenay). Il y a 6437 personnes qui habitent ces territoires $(2,3 \%$ de la région).

Profil $n^{\circ} 8$ (en dôme) : augmentation de la population suivie d'une baisse, avec un taux de variation négatif. Quatre municipalités font partie de ce type. Ici, il y a un changement de direction dans la courbe de la population, alors qu'un pic surgit au milieu de la période; la dernière partie montre une chute des effectifs. Deux municipalités vraiment périphériques appatiennent à ce groupe. Cependant, ce qui est étrange - mais pas surprenant —, c'est de voir apparaître Jonquière, un centre urbain dont l'importance des emplois industriels a décliné ces dernières décennies. On compte 22140 personnes en 2001 dans ce profil (22,1\% de la région).

Finalement, d'un côté, la carte montre beaucoup de nuances dans l'évolution de la population des municipalités de la région. D'un autre côté, elle permet de dégager un patron spatial fait de couronnes successives. Au milieu, il y a les centres urbains qui, après une croissance évidente, plafonnent (c'est le type 3); vient ensuite une première couronne composée d'espaces de banlieue et périurbains où la population crôit en flèche, surtout depuis les 20 dernières années (types 1,2 et 7); puis se dessine une deuxième et dernière couronne caractérisée par une baisse généralisée de la population et comportant des nuances tangibles (types 4, 5, 6 et 8).

\section{Graphique 1 - Diagrammes d'évolution, 1951-2001}
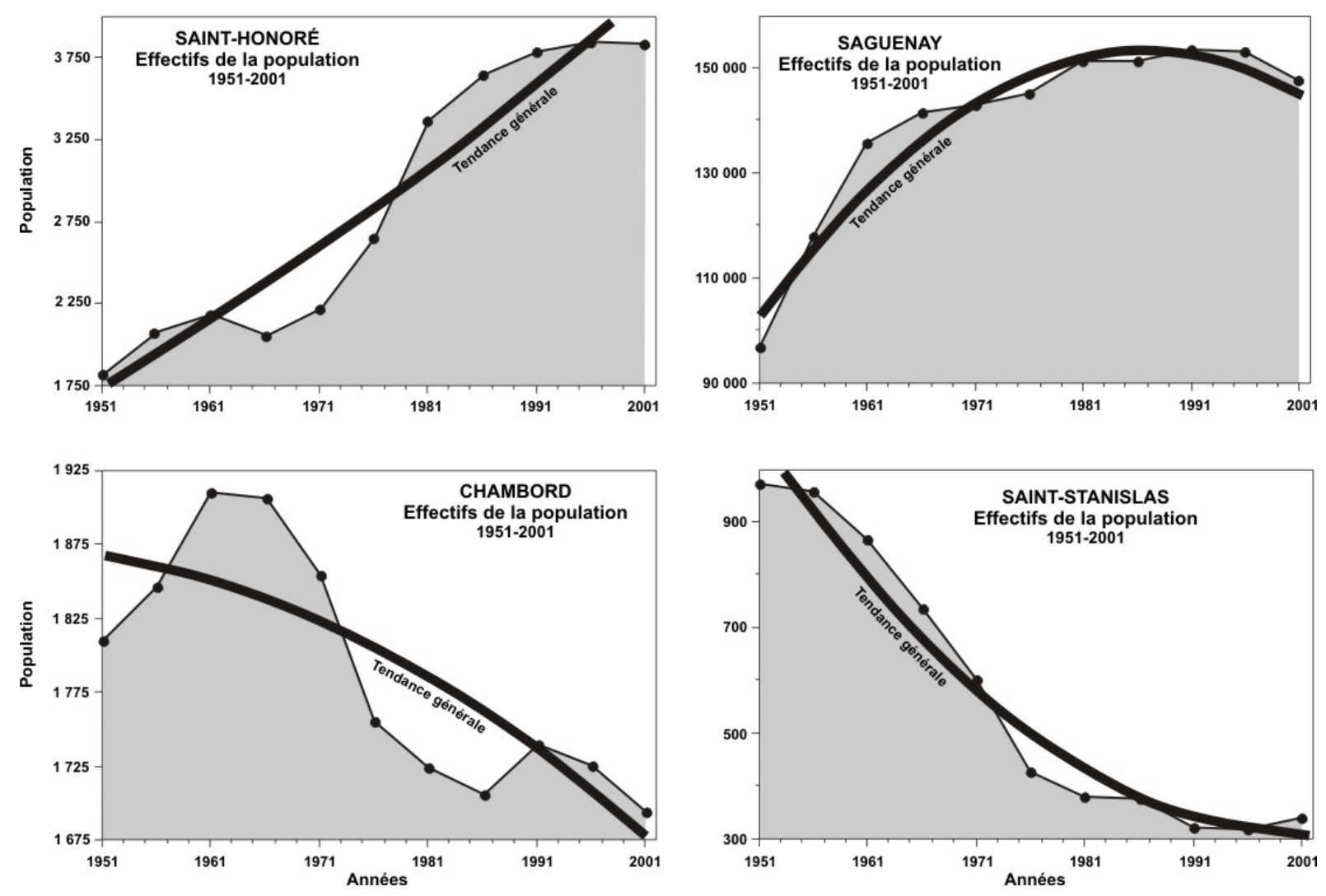


\section{Compléments d'explications}

Quelques éléments de nature historique permettent d'approfondir l'analyse des profils typologiques qui se dégagent de l'étude. On dirait qu'on assiste à un renversement de la spirale qui a présidé à la mise en place de la population. Les moins bons terroirs, les extrémités des percées du peuplement, là où l'écoumène était mité, les derniers espaces conquis, ont été les premiers à être l'objet du déclin de la population. Puis, à partir des années 1970-1980, la peau de chagrin se rétrécit au détriment des territoires qui ont fait partie de la seconde vague de peuplement. À ces références au temps se greffent et s'intègrent d'autres éléments.

\section{On dirait qu'on assiste à un renversement de la spirale qui a présidé à la mise en place de la population.}

Des explications sont d'ordre économique. Par exemple la distinction entre les profils $n^{\circ} 6$ (déclin ancien) et $n^{\circ} 4$ (déclin récent) n'est pas évidente, car ils occupent ensemble la marge de l'écoumène. Néanmoins, les hypothèses les plus plausibles seraient liées au peu de diversité dans les activités économiques et à une baisse fulgurante des activités agricoles pour les premières (profil $\mathrm{n}^{\circ}$ 6) (abandon des activités autarciques). Pendant ce temps, les emplois industriels se maintenaient dans les secondes (profil $n^{\circ} 4$ ). Depuis 1980, ce sont les secondes qui sont frappées par une baisse de la population en raison, en grande partie là aussi, du peu de diversification des activités, de la récession économique, du développement technologique, de la mondialisation, d'une concentration et d'une diminution des emplois industriels, notamment dans le secteur du bois (Marc-Urbain Proulx mentionne le nombre total de 4000 à 5000$)^{6}$.

D'autres dimensions explicatives sont davantage d'ordre politique. À ce propos, deux points méritent l'attention. Le plan agroforestier de 1971, dont le but était de régionaliser les productions agricoles, a confirmé une réalité d'inégalité spatiale et est venu accélérer le processus de déplacement des activités agricoles depuis la périphérie vers le centre et, partant, des populations ${ }^{7}$. À cela s'ajoute la campagne de reboisement des terres jugées peu propices à l'agriculture. Également, la loi du zonage agricole a sans doute eu des effets positifs pour contrer l'urbanisation mais, selon certains, elle a empêché l'installation de ruraux sur des lots dont seulement une partie est propice à l'agriculture ${ }^{8}$.

Des explications sont à caractère démographique. Il y a eu baisse générale de la natalité. On remarque, d'une part, des taux de masculinité élevés et une importance accrue des personnes âgées dans les territoires de la périphérie de la région et, d'autre part, une situation inverse dans quelques municipalités périurbaines, là où les jeunes couples sont proportionnellement plus nombreux. Il faudrait aussi compter l'exode de la population et surtout celle des jeunes.

Finalement, il est fortement recommandé de consulter les nombreuses cartes de l'Atlas électronique du Saguenay-Lac-Saint-Jean sur les modèles gravitationnels, les migrations de travail, la structure des âges et des sexes, la structure des emplois, l'utilisation du sol et bien d'autres pour tirer des explications et pour mieux comprendre la dynamique régionale ${ }^{9}$.

\section{Situation de Saguenay dans la dynamique}

\section{Bref historique}

Le territoire de la nouvelle ville fusionnée (2001) englobe les limites de nombreuses municipalités dont plusieurs désignations, de quartiers ou de secteurs, en conservent la mémoire. Avant les fusions des années 1970, plusieurs municipalités étaient contenues dans les frontières actuelles de Saguenay. Il n'y a pas si longtemps, il y avait des maires et des conseillers pour plus d'une douzaine de villes et de municipalités. Aujourd'hui, Saguenay regroupe les anciens centres urbains de Chicoutimi, Jonquière, La Baie et les territoires périurbains de Laterrière, Canton Tremblay, Shipshaw, Lac-Kénogami.

\section{Centre urbains et banlieue}

Le territoire de la ville de Saguenay mériterait une analyse avec un découpage moins grossier que celui des anciens territoires qui la composent, par exemple par secteurs de dénombrement ou îlots urbains. Il est malgré tout facile d'observer que les anciens centres urbains de Chicoutimi, de Jonquière et de La Baie commencent à voir décliner leur population au profit 
des territoires périurbain tels que Laterrière (maintenant dans la nouvelle ville) et Saint-Honoré, un village dortoir. On assiste ici à l'effet «trou de beigne », c'est-à-dire à un débordement vers l'extérieur au détriment du centre. Pour de plus amples informations, il est recommandé de consulter l'étude de Boivin sur l'évolution de l'utilisation du sol dans Saguenay de 1977 à $2001^{10}$.

\section{Place sur l'échiquier régional et provincial}

Le nombre de personnes qui habitent le territoire de Saguenay a augmenté au cours de la période étudiée; en nombre absolu, il part de 97044 hab. pour atteindre 148250 hab. avec un sommet de 153965 en 1991 (voir le tableau 1). Mais depuis les deux derniers recensements, on observe une chute non seulement du nombre de personnes, mais aussi de son poids relatif. Saguenay atténue sa position de leader au niveau de la région. En effet, même si l'on assiste à une augmentation générale de son poids démographique durant la période (de $49,30 \%$ à $53,27 \%$ ), il y a eu, depuis les 20 dernières années, une perte pondérale constante.

Ce qui est inquiétant également, c'est que le poids démographique de la ville et celui de la région décroissent dans l'ensemble québécois. Ainsi de 1961 à 2001 les pourcentages ont varié respectivement pour la ville et pour la région de $2,59 \%$ à $2,00 \%$ et de $4,99 \%$ à $3,75 \%$.

Tableau 1 - Situation de Saguenay, 1951-2002

\begin{tabular}{ccccc} 
Années & $\begin{array}{c}\text { Population : territoire } \\
\text { de Saguenay }\end{array}$ & $\begin{array}{c}\text { Ville de Saguenay / } \\
\text { région (\%) }\end{array}$ & $\begin{array}{c}\text { Région / Québec } \\
\mathbf{( \% )}\end{array}$ & $\begin{array}{c}\text { Ville de Saguenay / } \\
\text { Québec (\%) }\end{array}$ \\
1951 & 97044 & 49,03 & 4,88 & 2,39 \\
1956 & 118236 & 50,38 & 5,07 & 2,55 \\
1961 & 135984 & 51,82 & 4,99 & 2,59 \\
1966 & 141671 & 52,93 & 4,63 & 2,45 \\
1971 & 143313 & 53,95 & 4,41 & 2,38 \\
1976 & 145304 & 54,12 & 4,31 & 2,33 \\
1981 & 151776 & 54,23 & 4,35 & 2,36 \\
1986 & 151596 & 53,17 & 4,23 & 2,25 \\
1991 & 153965 & 53,80 & 4,05 & 2,18 \\
1996 & 153476 & 53,54 & 3,94 & 2,11 \\
2001 & 148250 & 53,27 & 3,75 & 2,00 \\
\hline
\end{tabular}

\section{Ce que réserve l'avenir}

L'analyse de l'évolution de la population révèle les tendances générales qui affectent les territoires municipaux. Il serait tentant de projeter les courbes vers l'avenir et de dire qu'ici, c'est prometteur et que là, c'est sans retour. C'est un exercice que nous ne ferons pas. Cependant, il y a des considérations qui méritent d'être soulignées.

Il est vrai que les effets de l'évolution de la population se font sentir dans toutes les collectivités. En dehors des sept centres urbains, trois situations bien distinctes apparaissent : 1) les territoires périurbains, en raison de leur croissance démographique, exigent de plus en plus de services et d'infrastructures (écoles, santé, rues et chemins); 2) les collectivités qui voient leur population décroître ces vingt dernières années sont et seront aux prises avec un surplus d'équipements et d'infrastructures qu'elles ne pourront plus faire fonctionner, qu'elles ne pourront plus se payer ; 3) puis, il y a les collectivités où le mal est fait. En effet, c'est là que le déclin de la population a commencé dès le début; déjà, au milieu des années 1970-1980, elles avaient atteint leur seuil le plus bas (quelques centaines). À ces endroits, plusieurs services n'existent plus : bureau de poste, épicerie, station d'essence et garage, caisse populaire, culte. Et même, il est devenu impossible de trouver des citoyens pour assurer la fonction de maire; c'est notamment le cas de Saint-Stanislas.

Quant à Saguenay, il faudra un bon coup de barre pour qu'elle assure son rôle de capitale régionale et qu'elle le renforce. Bien sûr, il n'y a pas uniquement les effectifs démographiques qui entrent en jeu dans le 
devenir d'une ville. Il y a aussi son dynamisme interne, ses forces économiques, sociales, environnementales et politiques. Elle est dépendante du dynamisme qui lui sera insufflé non seulement à l'intérieur de ses frontières, mais aussi et surtout dans la région tout entière.

\section{Conclusion}

Voilà un portrait quantitatif, clair, nuancé et condensé de l'évolution de la population dans les territoires locaux de la région du Saguenay-Lac-Saint-Jean. On a pu dégager des groupements de comportement (typologie) qui s'expliquent au plan historique et au plan de la géographie et de la socio-économie. La région du Saguenay-Lac-Saint-Jean, comme beaucoup de régions dites périphériques, perd du poids par rapport à l'ensemble du Québec, et Saguenay fait partie de cette dynamique. Cette nouvelle entité territoriale est censée être le moteur de la région, le centre des principales activités industrielles et du tertiaire. Mais elle tire de la patte. Cependant, sa position géographique au cœur d'une région, comme un moyeu où se rejoignent les rayons d'une roue, lui assure un rôle de moteur et d'animation sur le plan des communications et sur les plans économique, social et culturel ${ }^{11}$.

\section{La région du Saguenay-Lac-Saint-Jean, comme beaucoup de régions dites périphériques, perd du poids par rapport à l'ensemble du Québec.}

On a ainsi le droit de se poser des questions sur le devenir des populations, sur la qualité de vie dans les différents territoires de la région, sur les voies qui s'offrent pour un développement durable des collectivités. Il est à espérer que les lignes qui précèdent vont être utiles aux acteurs du développement et aux preneurs de décisions.

\section{Remerciements}

Nos remerciements aux partenaires du projet de l'Atlas électronique du Saguenay-Lac-Saint-Jean dont le CRCD-02, la Fondation de l'Université du Québec à Chicoutimi ainsi qu'à André Arsonneault pour son aide au plan statistique et à Martin Dion pour la structuration de la base de données.

\section{Notes et références}

1 Majella-J. Gauthier est géographe et professeur émérite à l'Université du Québec à Chicoutimi; il coordonne le projet de l'Atlas électronique du Saguenay-Lac-Saint-Jean. Éric Tremblay est historien-conservateur et agent de recherche à la Société historique du Saguenay. Carl Brisson est géographe et professionnel de recherche, également à l'Université du Québec à Chicoutimi.

2 Oris, Michel et al. (2000), Leviers d'une politique de développement durable : démographie, aménagement du territoire et développement durable de la société belge, Laboratoire de démographie, Université de Liège et Centre d'étude de gestion démographique pour les administrations publiques, $13 \mathrm{p}$.

Eggerickx, T. et al. (2002), Démographie et développement durable: migrations et fractures socio-démographiques en Wallonie 1990-2000, Liège, Université Louvainla-Neuve, $240 \mathrm{p}$.

3 Girard, Camil et Normand Perron, (1989), Histoire du Saguenay-Lac-Saint-Jean, Québec, Institut québécois de recherche sur la culture, coll. Les régions du Québec, $n^{0} 2$, $665 \mathrm{p}$.

4 Bertin, Jacques, (1967), Sémiologie graphique, Paris, Mouton, $431 \mathrm{p}$.

5 Laboratoire de recherche et d'expertise en télédétection et géomatique, Atlas électronique du Saguenay-Lac-SaintJean, Université du Québec à Chicoutimi,

www.uqac.ca/atlas.

6 Proulx, Marc-Urbain (2004). L'impasse actuelle de l'économie urbaine et régionale du Saguenay-Lac-Saint-Jean, communication livrée au colloque « Le devenir de Saguenay » tenu les 7 et 8 avril 2004 (www.uqac.ca/vsag2025).

7 Bolduc, Philippe et Jacques Tessier, (1971), Programme d'aménagement des ressources rurales du Saguenay-LacSaint-Jean, Office de planification et de développement du Québec, 195 p.

8 Vachon, Bernard, (2003), Le défi régional du gouvernement libéral, Montréal, www.uniovi.es/cecodet $/ \mathrm{mdl} /$ docu/vachon-le_defi_reg_du_gouv_lib.pdf, 6 p.

9 Gauthier, Majella-J., Roger Boivin, Carl Brisson, PierreMartin Côté et Martin Dion, (2001), Carte d'utilisation du sol de Ville de Saguenay de 2001, Université du Québec à Chicoutimi, texte d'accompagnement et carte, $23 \mathrm{p}$. Cédérom (PDF).

(Téléchargeable depuis www.uqac.ca/atlas).

10 Boivin, Roger, (2004), Évolution de l'utilisation du sol de Ville de Saguenay de 1977 à 2001, Université Laval, Département de géographie, mémoire d'activité de synthèse (réalisé en collaboration avec l'Atlas électronique du Saguenay-Lac-Saint-Jean), 213 p.

11 Gauthier, Majella-J. et Martin Dion, (2001), La dynamique géo-économique du Saguenay-Lac-Saint-Jean, Université de Sherbrooke, congrès de l'ACFAS, conférence. 


\title{
Publicité
}

\author{
Réseau VRM
}

